\title{
Optical Measurement Technique for Space Column Characterization
}

\author{
Danny A. Barrows*, Judith J. Watson ${ }^{\dagger}$, Alpheus W. Burner ${ }^{*}$ \\ NASA Langley Research Center, Hampton, VA 23681-2199 \\ James E. Phelps ${ }^{8}$ \\ Swales Aerospace, Hampton, VA 23681-2199
}

\begin{abstract}
A simple optical technique for the structural characterization of lightweight space columns is presented. The technique is useful for determining the coeficient of thermal expansion during cool down as well as the induced strain during tension and compression testing. The technique is based upon object-to-image plane scaling and does not require any photogrammetric calibrations or computations. Examples of the measurement of the coefficient of thermal expansion are presented for several lightweight space columns. Examples of strain measured during tension and compression testing are presented along with comparisons to results obtained with Linear Variable Differential Transformer (LVDT) position transducers.
\end{abstract}

\section{Introduction}

Ground based characterization studies are underway at NASA Langley Research Center for lightweight inflatable space columns under a program known as Enabling Concepts and Technologies for Large Space Structures. This program includes the development of advanced materials for large, ultra-lightweight deployable and inflatable space structures (Figure 1) that can be rigidized when put into service. Experimental and analytical investigations are necessary to characterize deployed/rigidized booms (columns) for future applications outside the Earth's atmosphere. One such assessment subjects the columns to various loads of compression and tension while in a cold $\left(-50^{\circ} \mathrm{C}\right)$ environmental chamber. As part of this characterization effort, a low cost optical technique that is relatively simple to implement has been developed that utilizes image processing and image scaling to measure the spatial displacements of discrete targets on the columns. These measurements are obtained without the complexity of photogrammetry. The displacements are then used to determine the deformation of the space columns, and hence the strain, in addition to the coefficient of thermal expansion (CTE).

\section{A. General Depiction of Columns}

\section{Hardware and Software Description}

Two different styles of a select group of approximately $10 \mathrm{ft}$ long columns have been studied and tested while subjected to extreme cold conditions as part of a tension/compression characterization effort. These columns are uniquely designed for specific applications of space related ultra-lightweight deployable space structures. Fabrication consists of proprietary composite thermoplastic materials suitable for the harsh and hostile conditions of low-earth orbit. The columns were sent to NASA from two different manufacturers fully deployed, ready for testing. Each column is individually packaged, labeled for identification, and removed one at a time from the packaging just prior to the beginning of each test.

\footnotetext{
* Aerospace Engineer, Advanced Sensing and Optical Measurement Branch, Member AlAA

${ }^{\dagger}$ Senior Research Scientist, Mechanics and Durability Branch, Senior Member AIAA

¥ Senior Research Scientist, Advanced Sensing and Optical Measurement Branch, Associate Fellow AIAA

${ }^{\S}$ Design Engineer, Swales Aerospace, Member AIAA
} 


\section{B. Environmental Test Chamber}

A unique specialized chamber, designed and constructed at Langley, is in use for testing lightweight columns in a controlled cold environment. The test chamber (Figure 2) is primarily constructed of an aluminum framework that supports three layers of Plexiglas walls. Its design houses a vertical test fixture used to apply loads to the columns, maintain a cold temperature environment, and control moisture buildup on the outside surfaces of the chamber. A load cell is mounted at the top of the fixture to measure the applied loads. The fixture is engineered to subject individual columns with compression and tension loads after being cooled to a uniform temperature of $-50^{\circ} \mathrm{C}$. A reversible hydraulic actuator is used to apply loads to the columns through the fixture from the bottom side of the chamber. Thermocouples are positioned on each column and throughout the chamber to monitor the temperature distributions for the duration of each test. Three LVDT (Linear Variable Differential Transformer) displacement transducers are evenly spaced at $120^{\circ}$ intervals around the circumference of each column. The \pm 0.1 -inch range LVDTs are used to record the differential contraction or expansion of the entire column using $10 \mathrm{ft}$ composite connecting rods during load testing at $-50^{\circ} \mathrm{C}$.

\section{Optical Measurement System}

Photogrammetry is typically the method of choice for measurement studies to determine static and dynamic profiling of inflatable and ultra-lightweight space structures. ${ }^{1}$ However, due to the intricacy in construction with the cold environmental test chamber used for this experiment, conventional photogrammetric techniques are not readily adaptable, partly due to the difficulty in achieving two or more views with sufficient camera separation to provide adequate measurement accuracy.

Real-time, non-intrusive target centroiding and tracking is used to establish the image plane coordinates as a function of time. A fairly straight forward technique is then used that converts pixel space to two-dimensional object space coordinates by means of a scaling multiplier calculated from in situ camera displacement calibrations along one dimension. A two-camera videogrammetric model deformation (VMD) measurement system serves as the image acquisition system for this study. ${ }^{2}$ The two-camera system, is however not being used in the conventional mode for which it was designed. Under normal operating conditions, the cameras simultaneously acquire images of a common area of the test article in order to determine three-dimensional spatial coordinates of the object space $(X, Y, Z)$ from two sets of image plane coordinates $(x, y){ }^{3}$ For these column measurements, rather than have the cameras look at identical locations they are positioned to look at two distinctly separate areas on a column (Figure 3) with a fairly large separation. This becomes two single-camera measurement systems in one convenient and synchronized package that yields an output set of centroids verses time for each camera.

\section{Test Setup}

Two progressive scan charge-coupled device (CCD) video cameras with $640 \mathrm{X} 480$ pixel resolution are rigidly mounted to individual translation stages (Figure 4) and bolted to a T-slotted modular frame (Figure 5) that is securely fastened to a massive support backstop. Each camera is equipped with a $70 \mathrm{~mm}$ focal length lens to provide high magnification at two separated areas of each column. The line of sight for each camera is set so the optical axis is approximately normal to one of the plexiglass walls on the test chamber in order to afford an optimum view of the targets on the test article and maximize the image displacement for a given object displacement. The video cameras are focused on given sets of column targets before initiating camera calibration. Calibrations are performed at the beginning of each new column test setup with the aid of digital displacement indicators, having precisions and certifiable accuracies of 0.0001 inch, to track camera movements primarily in the lengthwise direction of the column. These movements are only induced during the calibration procedure, not during column testing. At the end of each calibration the cameras are locked into position in order to accurately track and measure column displacement. Each column is tested inside the environmental chamber while securely mounted in precisely aligned fixtures. The fixtures are engineered to apply compression and tension loads to the columns after being cooled to a uniform temperature of $-50^{\circ} \mathrm{C}$. A single load cell is used to measure the applied loads, while three LVDT's ( \pm 0.1 -inch range) with composite connecting rods measure column displacements under load. Thermocouples are positioned at specific areas along the column and throughout the chamber to record temperatures during testing.

At the start of each test a select column is mounted in the test fixture of the environmental chamber and set up for compression or tension loading. No initial loading is applied to the installed column. The chamber is then sealed shut in preparation for the nitrogen purge, cool down and load test. During the purging process the incoming $\mathrm{N}_{2}$ and the air inside the chamber are vented outside to the atmosphere. This procedure takes about $60-90$ minutes to complete and is used to remove air moisture in the chamber prior to cool down. Temperatures are closely monitored 
throughout the chamber and on the column to ensure a uniform temperature distribution of $-50^{\circ} \mathrm{C}$ before testing commences.

\section{Optical System Calibration}

An image acquisition system is used to capture images from each of the two video cameras and display them in near real time on a graphical display monitor. Centroids of the targets on the columns are found and tracked using a threshold, flux-weighted algorithm. After initiating the target tracking sequence, in situ camera calibrations are performed by moving each camera in a vertical direction by known increments using a translation stage. This procedure simulates a column in motion with fixed cameras. The process is done in this manner because when a test column is securely mounted in the environmental chamber test fixtures displacement of the column is not possible for calibration.

Targets are affixed to the test column before being installed in the environmental chamber fixtures. The target positions are measured with respect to a reference point at the end of the column. These measurements are made using an optical measurement comparator and a calibrated 48-inch precision scale. A general layout of the targeting scheme can be seen in figure 6. The targets are painted on the columns as small dots using a small applicator. Colors are dictated by the configuration of the column being studied. Ideally, a significant contrast in grayscale is desired such as white-on-black or black-on-white. The diameters of the circular targets are approximately $0.06 \mathrm{inch}$. Distances between targets in each group are approximately 0.25 inch while the displacement between target groups is 41.5 inches. The high local precision coupled with the large spatial separation of targets imaged separately by the two cameras leads to reasonable precision in CTE and strain computations. The small displacements measured for each camera over a large distance between cameras increases the potential for high relative precision.

The cameras are repositioned in the lengthwise direction of the column by known incremental amounts of 0.05 -inch, up to 1.00 -inch overali, using the transiation stages. Target centroid data is recorded by the video cameras in two dimensional pixel space. Actual camera calibration reduction procedures consist of a basic linear least squares estimation of the slope of the change in pixels versus the known incremental camera displacements. An inch-per-pixel scaling multiplier is thus obtained along with a precision estimate of this calibration parameter by least squares. Residuals from the calibration procedure are typically below 0.001 inch (Figure 7) with the standard deviation of the residuals about one-half that value. Since the precision in object space depends on the field of view, even better precision is possible by further limiting the field of view.

Although the image plane scaling in the transverse direction to the length of the column was not measured, the pixel scaling factor in that direction should be nearly identical to the vertical scaling factor. The camera used has square pixels and the cameras were aligned approximately normal to the column. Thus, the image-to-object plane scaling is approximately the same in the transverse direction as lengthwise. Another issue is the undetected out-ofplane motion that can lead to inaccuracies in the technique, which may be improved by the use of telecentric lenses.

\section{Optical Measurement and Column Load Tests}

The pixel scaling technique developed for this work is the primary displacement measurement technique used during the cool down process. The LVDTs are only used during load testing and not during cool down. The two video cameras employed for this process each concentrate on a set of column targets that are separated by approximately 42 inches. A target set consists of five targets each, with the set near the top of the column being targets 1-5 and the set closer to the bottom of the column being targets 6-10. Individual tests begin at ambient temperatures and are cycled through a nitrogen gas purge before being cooled to a temperature of $-50^{\circ} \mathrm{C}$ using $\mathrm{LN}_{2}$. Complete time histories of measured target displacements on a column are recorded and analyzed at a continuous data-sampling rate of $1.6 \mathrm{~Hz}$ (Figure 8). An entire cool-down cycle can take up to 6 hours to complete. Actual load tests on the columns begin at the completion of this cool-down process. A reversible hydraulic actuator on the test fixture is used to apply forces to the columns. The direction of the actuator's motion depends on the type of load being applied, either in compression or in tension. These forces are applied to the columns only during the test portion of program cycle, not during the calibration, purge, or cool down. A load test begins when the thermocouples distributed throughout the chamber, including those monitoring the temperature of the column being tested, reach a sustained $-50^{\circ} \mathrm{C}$. At such time the actuator induces a total force of no more than $75 \mathrm{lbf}$ to the column at a rate of about $20 \mathrm{lbf}$ per minute. The maximum applied force is held constant for about one minute then released. The column is allowed several minutes to settle back into its original position before the process is repeated. A minimum of three load tests is performed on each column per program cycle. At the completion of the testing cycle, the flow of $\mathrm{LN}_{2}$ is removed and the system is allowed to return to room temperature. 


\section{Data Reduction and Experimental Results}

Centroid tracking is used to establish image plane coordinates during cool down and load testing. The scaling multiplier calculated from camera calibrations prior to the start of each test is used to convert the image coordinates in pixels to fractional inches of displacement. During the nitrogen purge portion of a test cycle temperatures inside the environmental chamber increase by a small amount due to warming gas. The target tracking data in figure 8 is for one camera only and shows the representative behavior of one of the target areas during purge, cool down, and test. The overall column behavior is computed by subtraction of the independent results from each camera. When $\mathrm{LN}_{2}$ is introduced to the system for cool down, the column length decreases due to thermal contraction. As the temperatures inside the chamber begin to equalize at the required $-50^{\circ} \mathrm{C}$ set point, column contractions begin to ease, the system stabilizes, and the test advances to the load-testing portion of the program cycle.

\section{A. Applied loads and column displacements}

Load testing on the columns typically begin and end at a steady rate of about $20 \mathrm{lbf}$ per minute. An applied force, either in compression or in tension, is closely monitored until a maximum total load of $75 \mathrm{lbf}$ is met. After about one minute, the applied load is released and the process is repeated at least two more times. Figure 9 provides an example of a displacement-verses-time profile along the column length (vertical) and the traverse (perpendicular to column length) movement during these conditions. Three individually tested tension loads are illustrated in the figure showing vertical and horizontal column target displacements for all of the targets on the column. Measurements in vertical length range from 0.0013 to 0.0023 inches while horizontal target displacements range from 0.0030 to 0.0088 inches. In many cases the horizontal column movements are greater than or equal to vertical movements during all of the tension tests performed. The displacements in the horizontal are slightly larger than those in the vertical for several reasons. The columns may be rotating very slightly in the fixture as the forces are applied. The targets may also be shifting in the horizontal due to small stretching or realigning of the column as the column tries to straighten out, again from loading. Some of the much larger horizontal shifts ( 0.015 to 0.040 inches) seen in figure 9 are likely coming from physical adjustments that are made to the fixture's hydraulic load ram between load tests. When re-referencing the column targets to zero at the start of the three load tests for this column the dimensional spread between targets increases slightly, particularly for targets 6 through 10 in tests 2 . This effect could be from the repositioning of the column during the hydraulic ram adjustment. The data in figure 9 is intended to show the amount of total column target movement relative to the start of the first test. The data is normally rereferenced to zero at the start of each loading test.

\section{B. Determining the coefficient of thermal expansion CTE during cool down}

The displacements between two target sets on a column represent a change in length for that portion of the column. Figure 10 summarizes the changes made in the vertical and horizontal over a 41.5 -inch span for a column through cool down and a tension test. Displacements between targets sets are determined as the differences between target sets, for instance ( 1 and 6), ( 2 and 7), ( 3 and 8), etc, (figure 6). In preparation for a tension test, a column is suspended from the top of the loading fixture inside the cold box chamber during the nitrogen gas purge. The bottom of the column is allowed to slide freely on a closely fitted dowel shaft that is also part of the loading fixture. The temperatures inside the chamber increase a moderate amount during the system purge, as does the length of the column. As the column grows ever so slightly, so does the distance between target sets. The same is true when cooling with $\mathrm{LN}_{2}$, except then the temperatures are dropping and the column is contacting. The coefficient of thermal expansion CTE can be computed from the relative contraction starting at an ambient condition to the minimum obtained temperature $\mathrm{T}$. The following expression is used to calculate CTE,

$$
\mathrm{CTE}=(\Delta \mathrm{L} / \mathrm{L}) / \Delta \mathrm{T}
$$

where, $\mathrm{L}$ is the length between target sets, $\Delta \mathrm{L}$ is the change in length between target sets, and $\Delta \mathrm{T}$ is the change in temperature. Table 1 shows the CTE results obtained for several columns.

A plot of the relative contraction $\Delta \mathrm{L} / \mathrm{L}$ as a function of temperature as a column is cooled down from ambient to $-50^{\circ} \mathrm{C}$ is presented in figure 11 . The relative contraction is determined as the mean of the differences in displacement of 9413 data points consisting of 2 sets of 5 -targets each, separated by 41.5 inches. The slope of the relative contraction versus temperature is noted to be the CTE provided it is independent of temperature. For the column contraction data presented in figure 11 the slope, and hence CTE, was computed from linear least squares to be $1.60 \times 10^{-6}{ }^{\circ} \mathrm{C}^{-1}$. The standard deviation of the slope (CTE) computed from least squares of $0.0017 \times 10^{-6}{ }^{\circ} \mathrm{C}^{-1}$ corresponds to about $6 \mu$ inches of contraction variability for the data of figure 11. The correlation coefficient was 
determined to be 0.9944 indicating a linear relationship. Note that the final difference between the displacements of the 2 sets of targets separated by 41.5 inches is only 0.0056 inch after the cool down $\Delta \mathrm{T}$ of about $-85^{\circ} \mathrm{C}$.

\section{Horizontal Motion Effect}

Vertical and horizontal column movements recorded during three sequential tension load tests for a single column can be seen in figure 12. The total vertical displacement between target sets involves a growth of less than 0.001 inch for each. The horizontal motion is larger ( 0.005 inches) at the beginning of each load test, and is then reduced as each test progresses. The distance formula can be used to calculate the total displacement along the two axes of deformation, but the third dimension is unknown since it was not measured. Ideally, additional cameras viewing the columns at $90^{\circ}$ (relative to the current camera locations) could help remove some of the uncertainties in calculating the total displacements between target sets and help in giving a clearer 3D perspective of how the columns are deforming. However, because of the 41.5 -inch length between target sets only small cosine errors are introduced. For example, using the Pythagorean Theorem, an apparent 41.5 -inch length changes by only 0.00012 inch when a target set is displaced horizontally by 0.100 inches. Due to limited access around the workspace of the cold box chamber additional cameras are difficult to install.

\section{Temperature Profile}

All of the columns involved in these series of tests are very lightweight with little mass. Column temperatures fall quickly when $\mathrm{LN}_{2}$ is allowed to flow into the chamber. There are numerous thermocouples distributed throughout the chamber and on the column being evaluated. Figure 13 demonstrates a typical comparison between temperature profiles and column contractions during a cool down. Thermocouples are located $90^{\circ}$ degrees apart at the top and bottom, $25 \%, 50 \%$, and $75 \%$ positions along the length of the column. The load tests promptly begin when temperatures settie at the required $-50^{\circ} \mathrm{C}$, approximately 3-1/2 to 4 hours after the beginning of the system purge. Temperatures are held as close as possible to $-50^{\circ} \mathrm{C}$ through the duration of the load tests, which takes approximately 15 to 20 minutes to complete. Once testing sequences are complete, the $\mathrm{LN}_{2}$ is removed, and the column and cold box chamber are allowed to naturally warm back to room temperature.

\section{E. Measurement of Strain}

The primary measurement of strain, or elongation per unit length, of the columns undergoing tension and compression were based on 3 LVDTs with a base separation of 118 inches. The optical measurement technique was also used for these strain measurements and is compared to the strain measurements recorded by the LVDTs. These results are presented in table 2 and figure 14 for a column during tension testing and in table 3 for a column during compression testing, both conducted at a temperature of $-50^{\circ} \mathrm{C}$. For both the LVDT and video data the slope of strain versus load was computed from a linear least squares fit. This slope is proportional to the inverse of the Young's modulus. For the video data the elongation was determined as the mean of the differences in displacement of 2 sets of 5-targets each, separated by 41.5 inches. Table 2 and Table 3 presents the predicted strain (in units of $\mu$ strain) at a load of $70 \mathrm{lbf}$ based on the strain versus load slope for 3 repeat tension and compression tests respectively of the same column. The strain data is seen to generally fall within the minimum and maximum of the LVDT strain data for the tension test, but with larger data scatter than the LVDT results. The data scatter for the compression test was over 2 times greater than for the tension test. A comparison of the data scatter as a function of load for a single tension test is presented in figure 14. The data scatter is significantly more than the LVDT data, but the general linear trends reflecting Hooke's Law are evident in both the video and LVDT data. The scatter in the video data could be reduced by increasing the separation of the target sets used to determine the elongation. In general the scatter in the data is inversely proportional to the separation of the target sets. Generally the video data were found to lie within the extremes of the LVDT data. It is best to use the y-intercept to zero the video data representing strain verses load. The slope of strain versus load is the primary reporting metric of the video technique.

\section{Other Applications}

Optical measurement techniques such as that used for this column characterization study can be beneficial for other one-dimensional or two-dimensional measurement applications. For example, the technique was recently used to determine detailed displacement measurements along the length of a model support sting at the NASA Langley Transonic Dynamics Wind Tunnel (TDT) while under static load. Information on the nature of sting bending is required for specific model testing at the TDT and is of interest for general facility characterization. The same twocamera measurement system used for this column characterization was supplemented with two digital indicators to 
perform in situ camera calibrations and verifications of the vertical and horizontal sting bending for static loads up to $600 \mathrm{lbs}$ during a series of wind-off tests.

\section{Concluding Remarks}

The low-cost optical measurement technique described in this report has a potential for producing high precision displacement measurements without the overhead of photogrammetric calculations. When utilizing a high magnification lens on a digital camera, accurate sub-pixel motions can be detected and measured. As magnification increases so does the potential for high precision. However, undetected out-of-plane motion can lead to inaccuracies in the technique, which may be improved by the use of telecentric lenses. Also if significant 2-D motion is expected, then it may become necessary to perform a two-dimensional in situ camera calibration when using this optical measurement technique.

The technique has been found to be very useful for characterizing lightweight inflatable space columns during ground tests. Coefficients of thermal expansion during cool down have been calculated for these columns along with induced strain calculations during tension and compression testing. A single-camera, single-view image scaling technique has been implemented to account for the poor accessibility to utilize photogrammetric techniques. Further empirical research is necessary to gain a better insight and understanding of this optical measurement technique.

\section{References}

${ }^{1}$ Adrian A. Dorrington, Thomas W. Jones, Paul M. Danehy and Richard S. Pappa, "Laser-Induced Fluorescence Photogrammetry for Dynamic Characterization of Transparent and Aluminized Membrane Structures", $39^{\text {th }}$ AIAA/ASME/SAE/ASEE Joint Propulsion Conference, Huntsville, Alabama, AIAA 2003-4798, July 20-23, 2003.

${ }^{2}$ Tianshu Liu, Sanjay Garg. Video Model Deformation System. User's Guide Ver. 1.1. High Technology Corporation: 2000.

${ }^{3}$ A.W.Burner and Tianshu Liu, "Videogrammetric Model Deformation Measurement Technique", Journal of Aircraft, vol. 38, no. 4, July/August 2001, pp. 745-754. 


\begin{tabular}{|c|c|c|}
\hline \multicolumn{3}{|c|}{ Measured CTE, $1{ }^{\circ} \mathbf{C}$} \\
\hline & & \\
Specimen No. & Column Type A & Column Type B \\
\hline 1 & $2.6 \times 10^{-6}$ & $0.9 \times 10^{-6}$ \\
2 & $2.7 \times 10^{-6}$ & $0.8 \times 10^{-6}$ \\
3 & $2.6 \times 10^{-6}$ & $1.1 \times 10^{-6}$ \\
4 & $2.3 \times 10^{-6}$ & $1.2 \times 10^{-6}$ \\
5 & $1.9 \times 10^{-6}$ & $0.9 \times 10^{-6}$ \\
\hline Mean & $\mathbf{2 . 4 \times 1 0 ^ { - 6 }}$ & $\mathbf{1 . 0 \times 1 0 ^ { - 6 }}$ \\
\hline
\end{tabular}

Table 1. CTE (Coefficient of Thermal Expansion) calculations for two different style columns from ambient temperatures to $-50^{\circ} \mathrm{C}$.

\begin{tabular}{|c|c|c|c|c|c|}
\hline & LVDTmin & LVDTmax & LVDTavg & Video & \% Diff. \\
\hline test 1 & 17.67 & 22.16 & 19.73 & 19.71 & -0.1 \\
\hline test 2 & 17.92 & 21.44 & 19.61 & 17.54 & -10.6 \\
\hline test 3 & 17.63 & 22.32 & 19.93 & 18.99 & -4.7 \\
\hline range & 0.29 & 0.88 & 0.32 & 2.17 & 578 \\
\hline
\end{tabular}

Table 2. Comparison of minimum, maximum, and mean LVDT and video determinations of $\mu$ strain at a $70 \mathrm{lbf}$ load column (based on slope of $\mu$ strain versus load) for 3 repeat tension tests of the same column at $-50^{\circ} \mathrm{C}$.

\begin{tabular}{|c|c|c|c|c|c|}
\hline & LVDTmin & LVDTmax & LVDTavg & Video & \% Diff. \\
\hline test 1 & 18.04 & 23.54 & 20.44 & 17.83 & -12.8 \\
\hline test 2 & 17.73 & 22.00 & 19.49 & 14.15 & -27.4 \\
\hline test 3 & 18.27 & 23.67 & 20.69 & 19.65 & -5.0 \\
\hline range & 0.54 & 1.67 & 1.20 & 5.50 & 358 \\
\hline
\end{tabular}

(units $=\mu$ strain)

Table 3. Comparison of minimum, maximum, and mean LVDT and video determinations of $\mu$ strain at a $70 \mathrm{lbf}$ load (based on slope of $\mu$ strain versus load) for 3 repeat compression tests of the same column $-50^{\circ} \mathrm{C}$. 

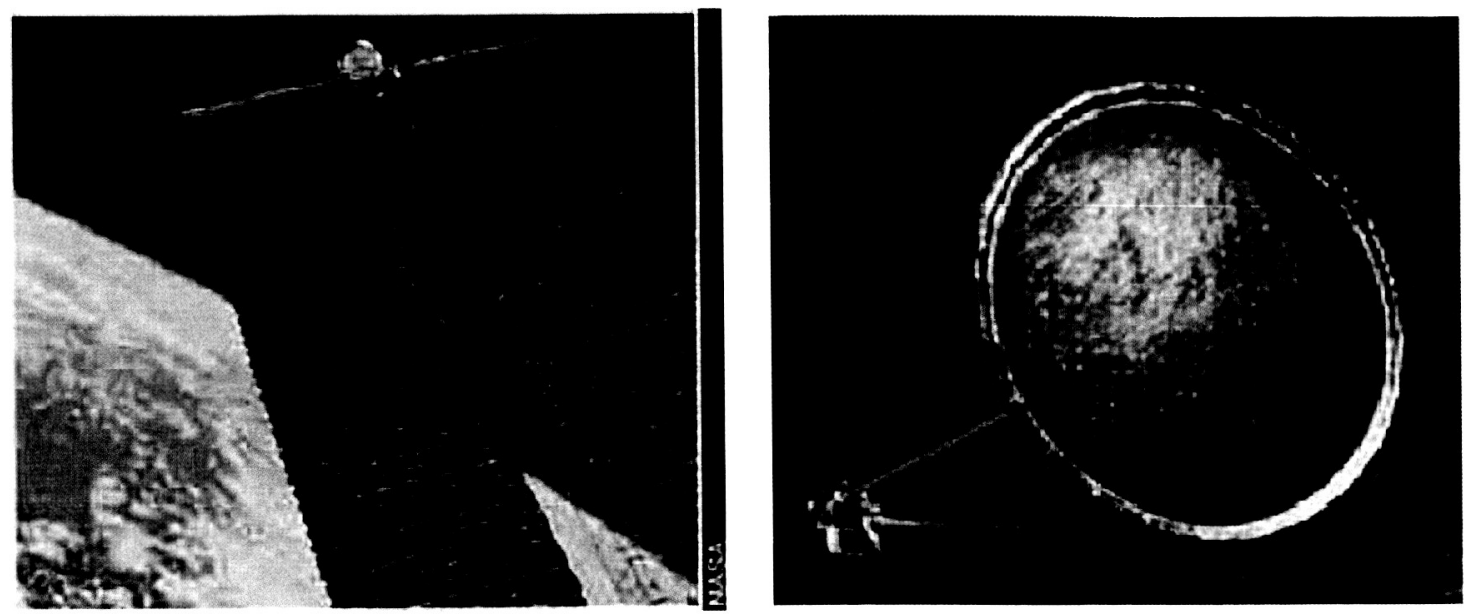

Figure 1. Large solar panel space structures.

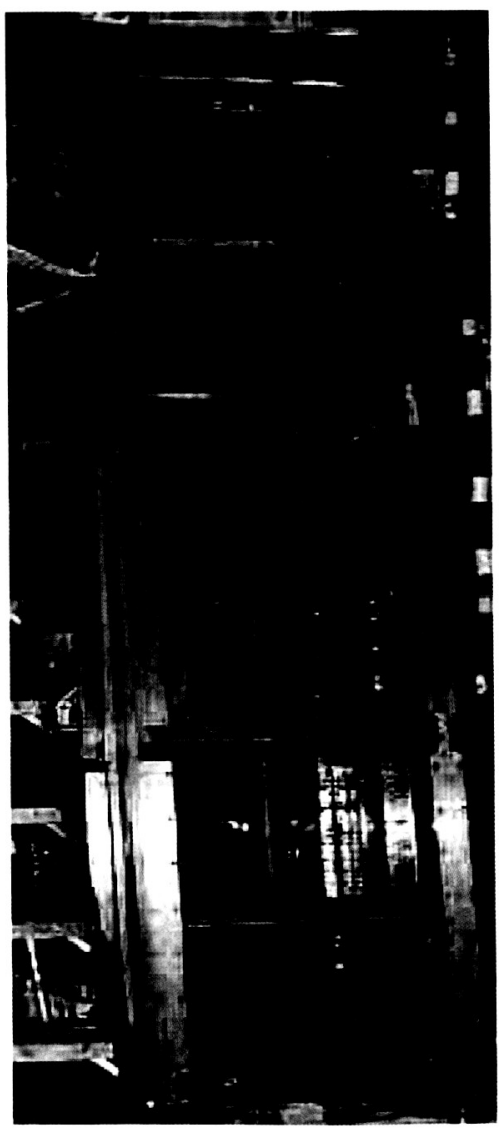

Figure 2. Thermal test chamber. 


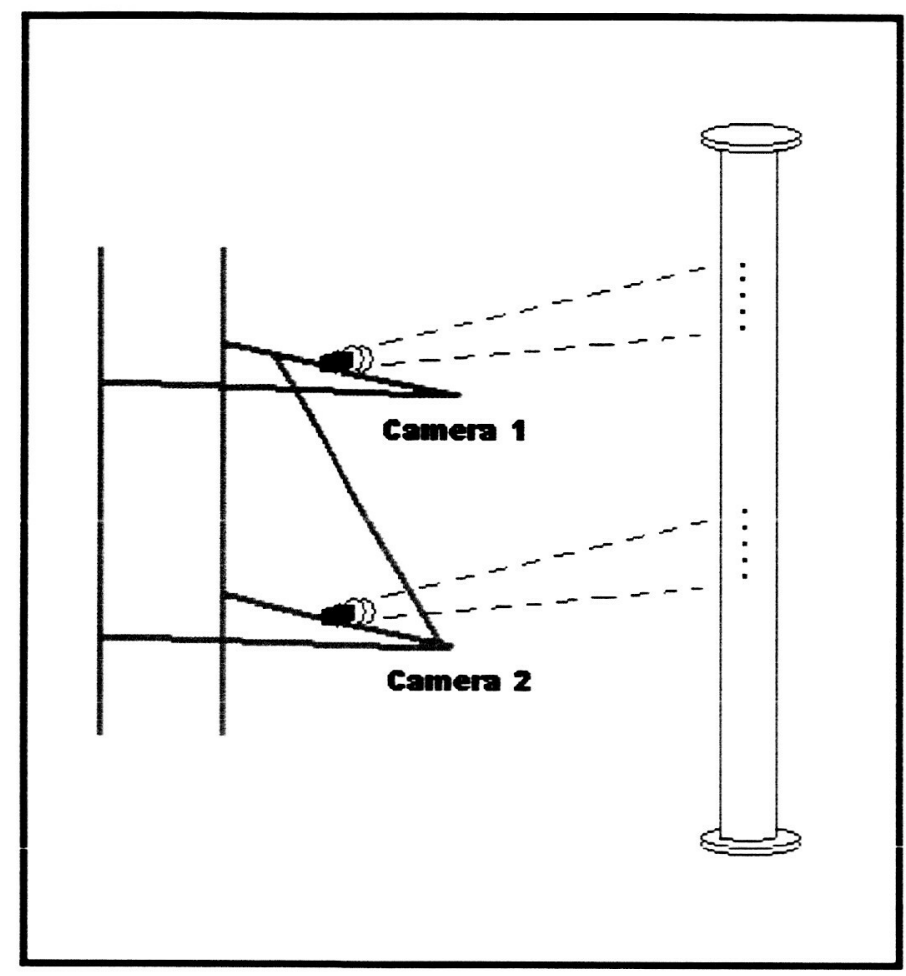

Figure 3. Camera positions with respect to targets on the columns.

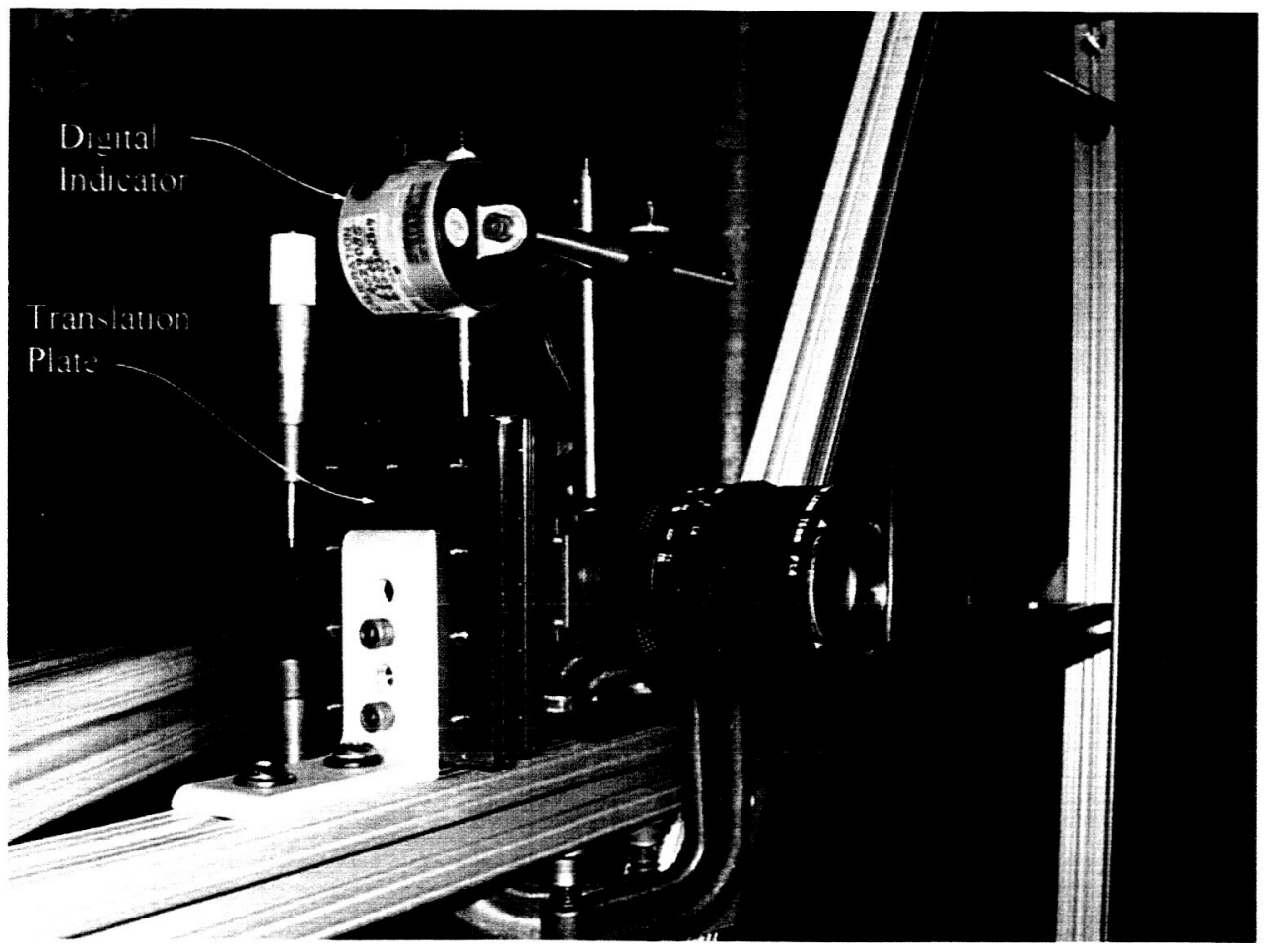

Figure 4. CCD video camera and supporting hardware setup. 


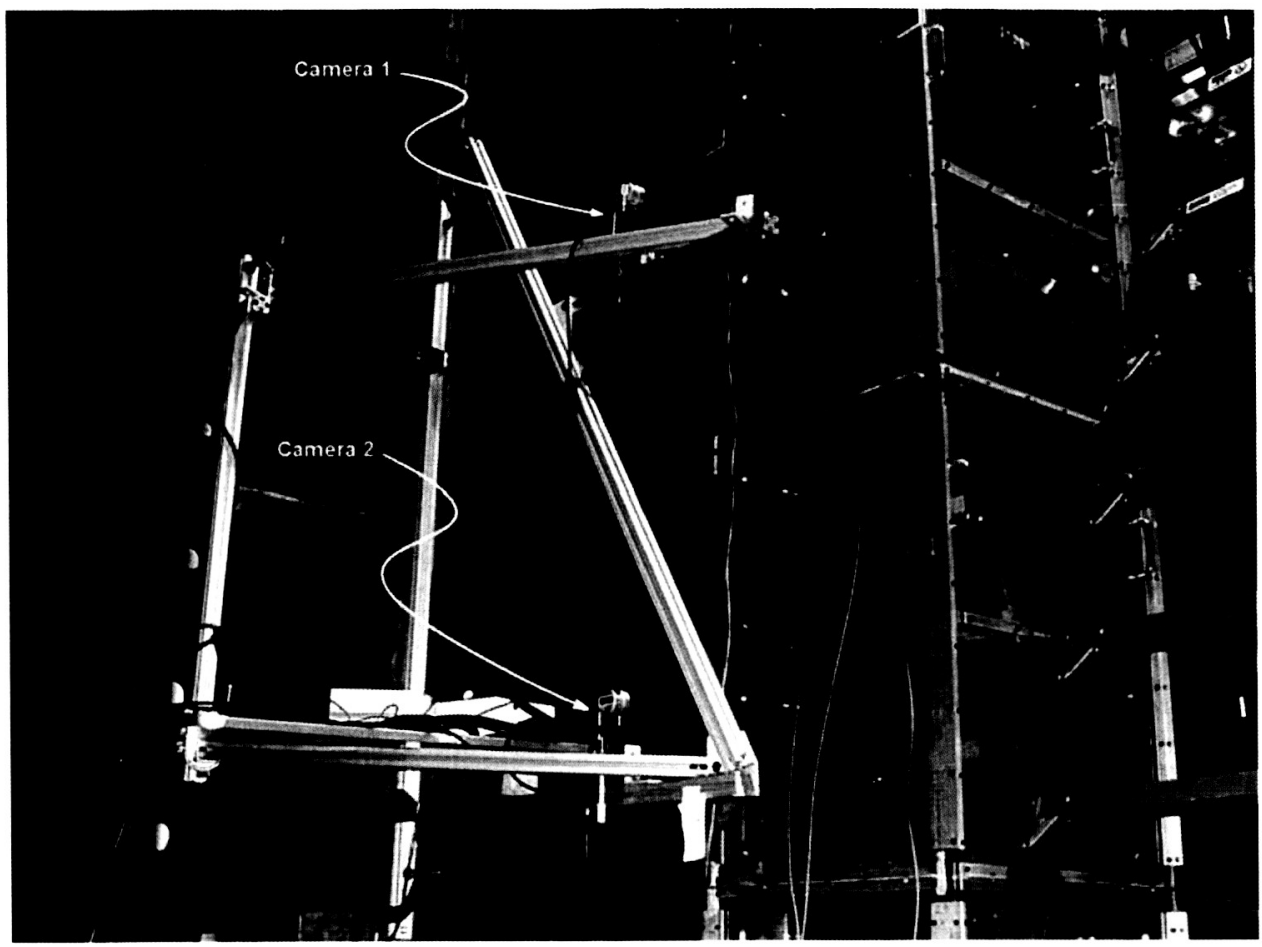

Figure 5. Camera mounting framework and test chamber.

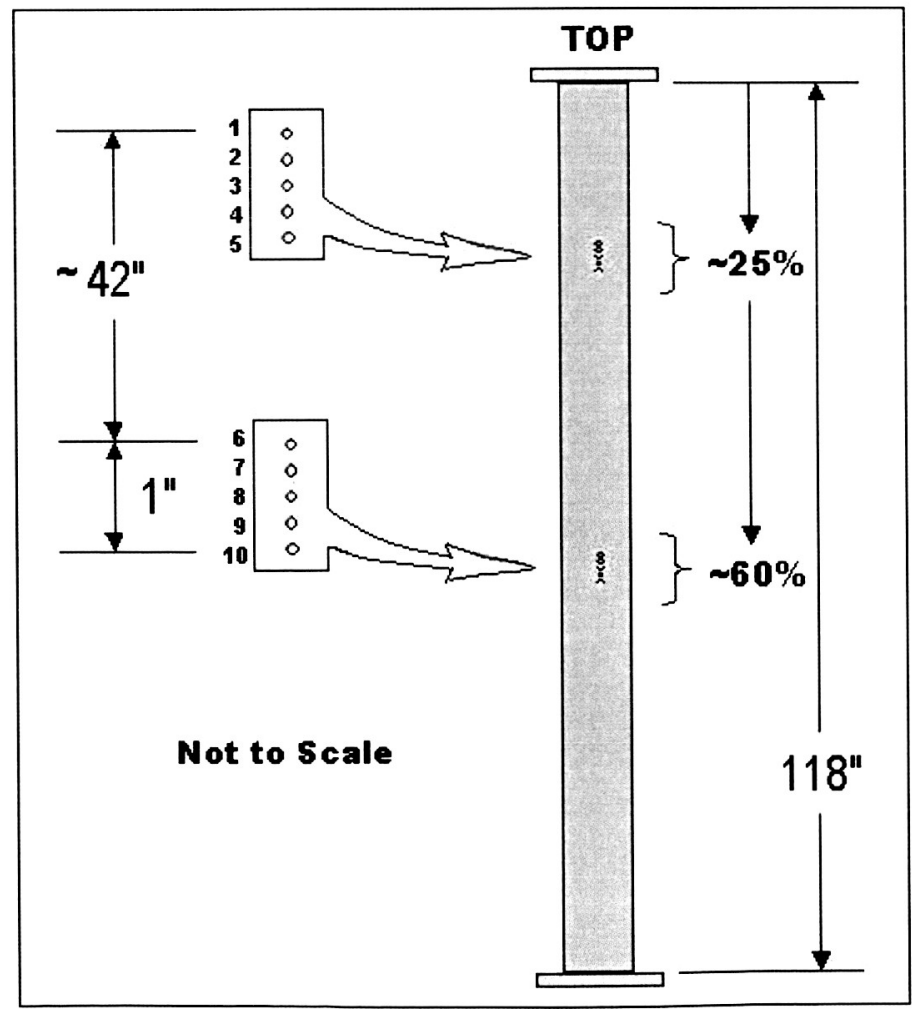

Figure 6. Example layout for the targets on a column. 


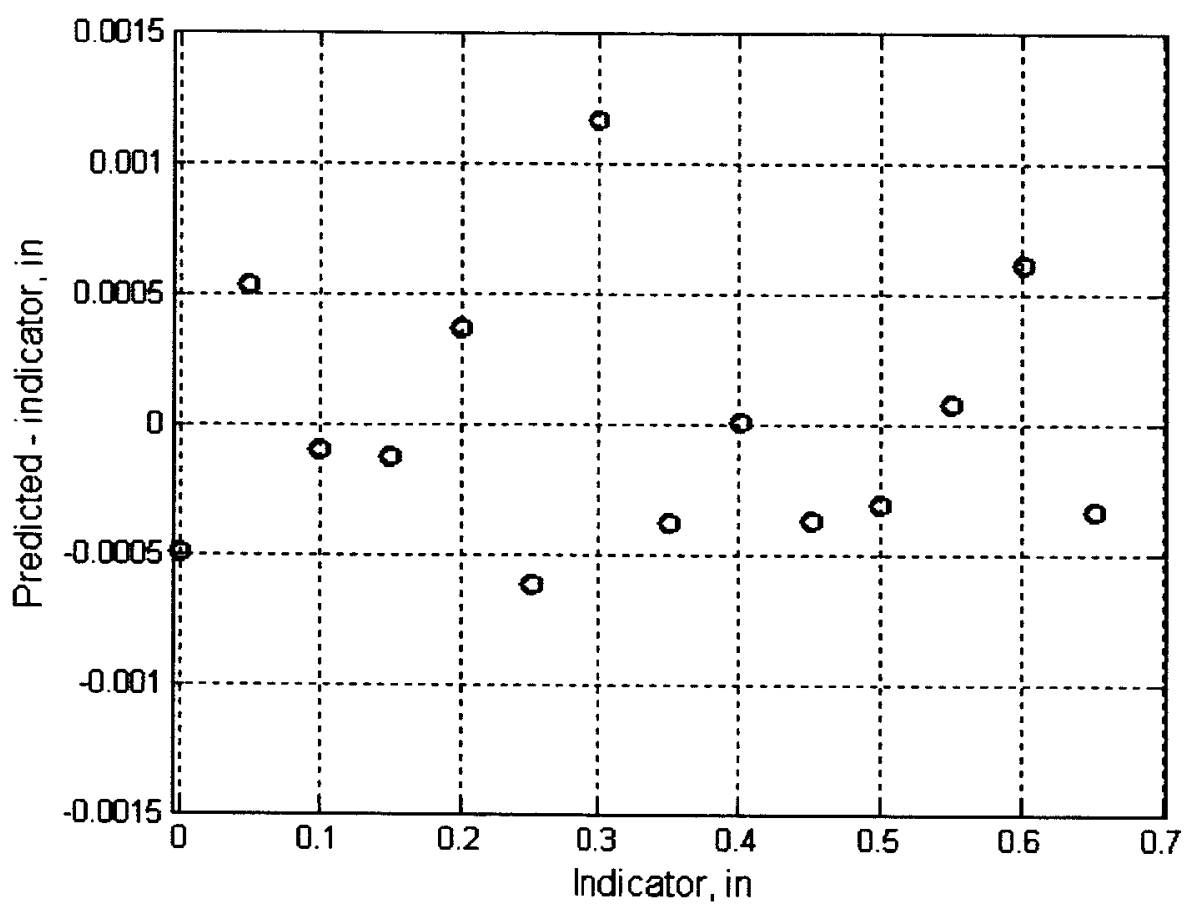

Figure 7. Example of residual error during a camera calibration.

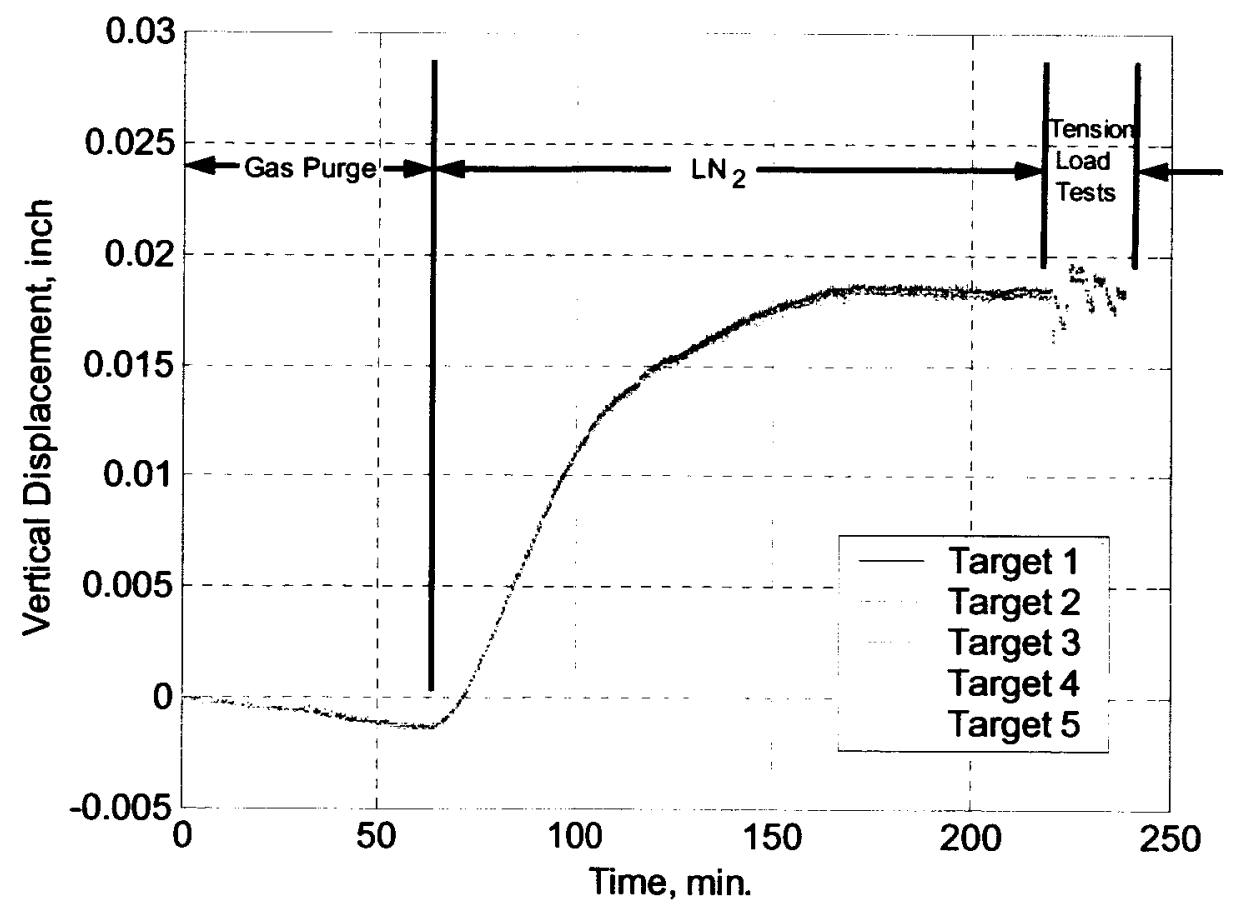

Figure 8. Complete test cycle - Purge, $\mathrm{LN}_{2}$ Cool Down, and Load Test. 

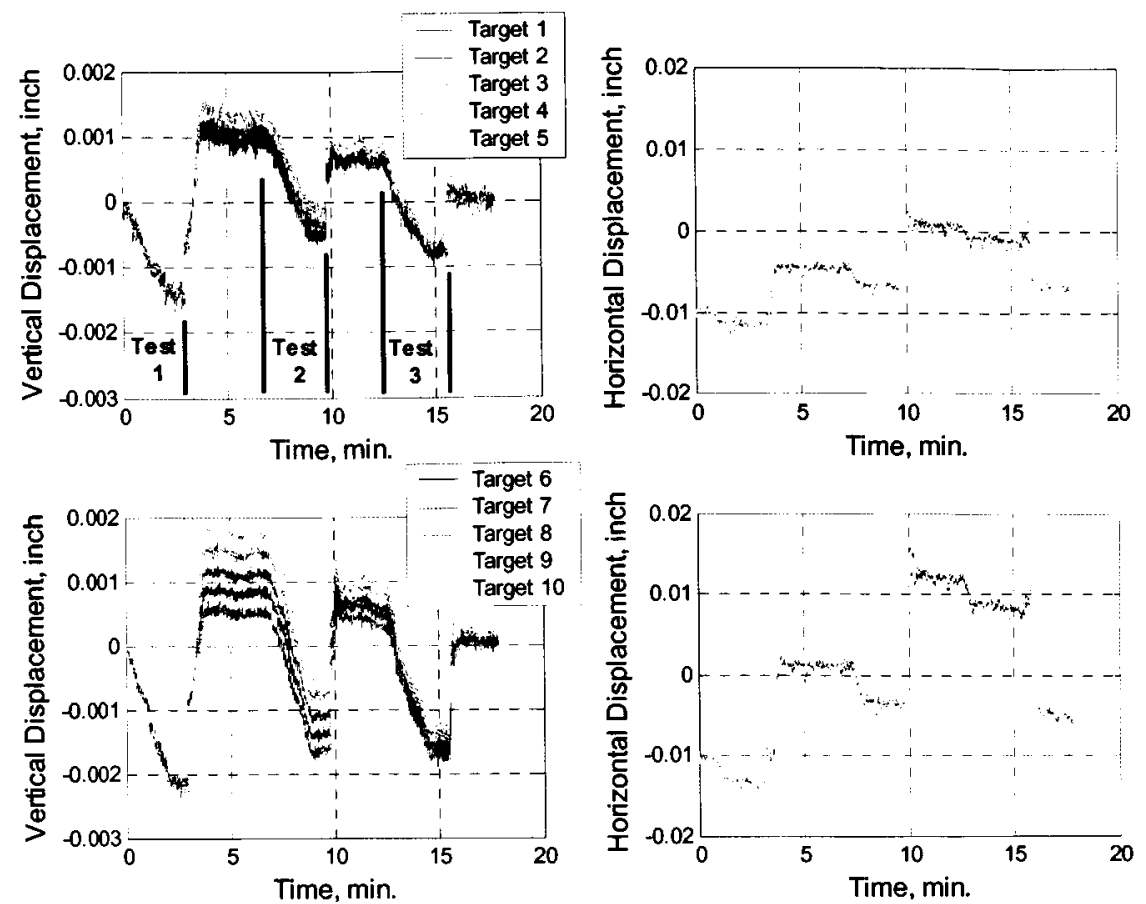

Figure 9. Target displacements during a tension load test. Targets $1-5$ are approximately $25 \%$ down from the top of the column (6-10 approx. $60 \%$ down).
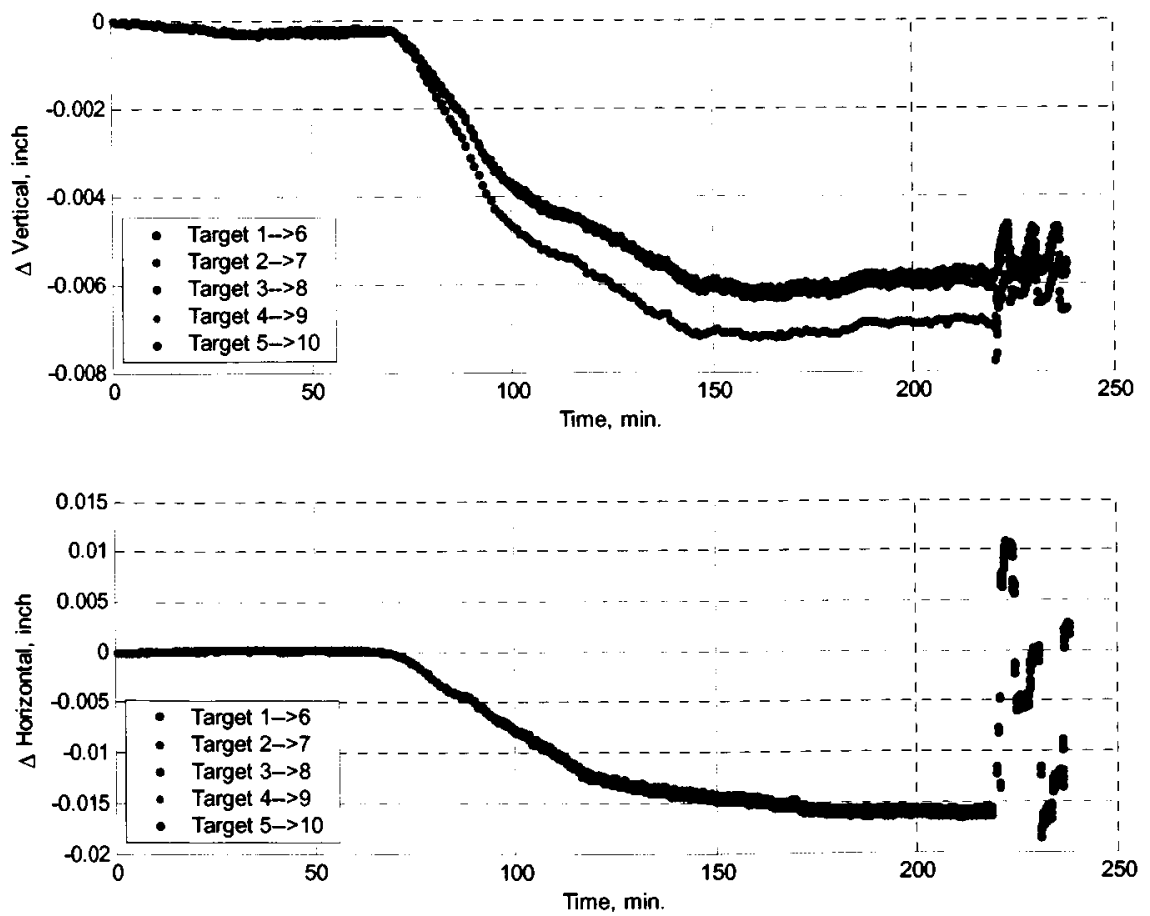

Figure 10. Total vertical and horizontal target displacement between target sets ( 1 thru 5$)$ and (6 thru 10) at 41.5 inch separation during cool down $\left(23^{\circ} \mathrm{C}\right.$ to $\left.-50^{\circ} \mathrm{C}\right)$. 


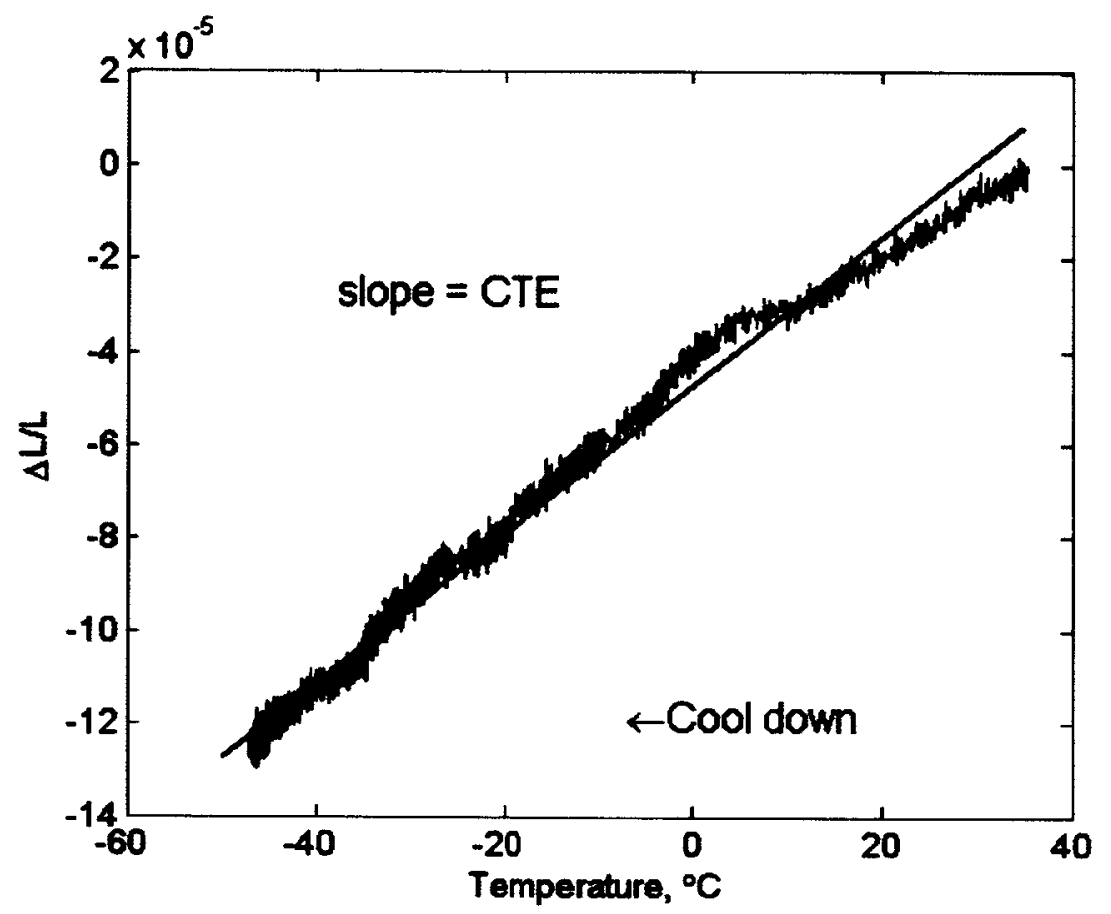

Figure 11. Relative contraction $\Delta \mathrm{L} / \mathrm{L}$ measured with the video technique with determination of the coefficient of thermal expansion $\mathrm{CTE}$ as a column is cooled down from $+35^{\circ} \mathrm{C}$ to $-50^{\circ} \mathrm{C}$.
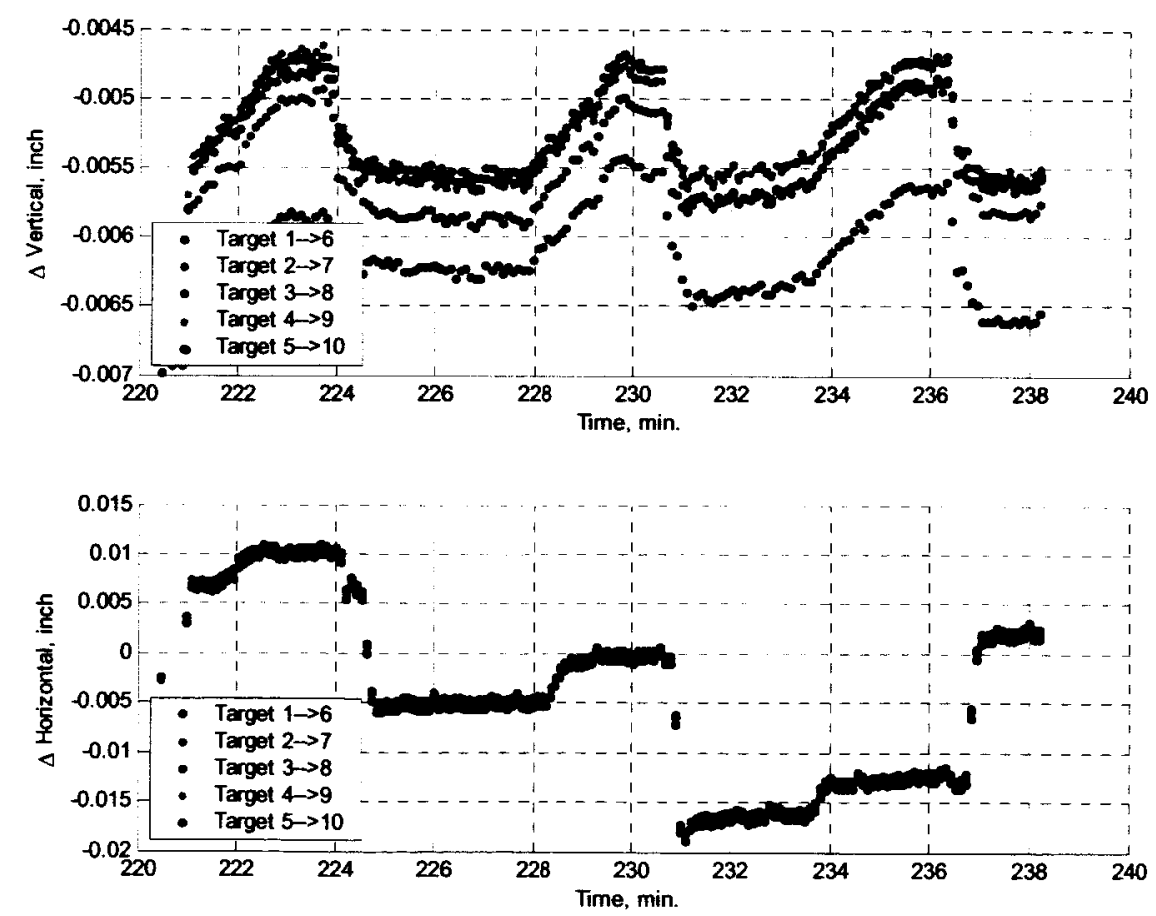

Figure 12. Total vertical and horizontal target displacement between target sets ( 1 thru 5) and (6 thru 10) at 41.5-inch separation during a tension load $\left(\right.$ at $\left.-50^{\circ} \mathrm{C}\right)$.

American Institute of Aeronautics and Astronautics 

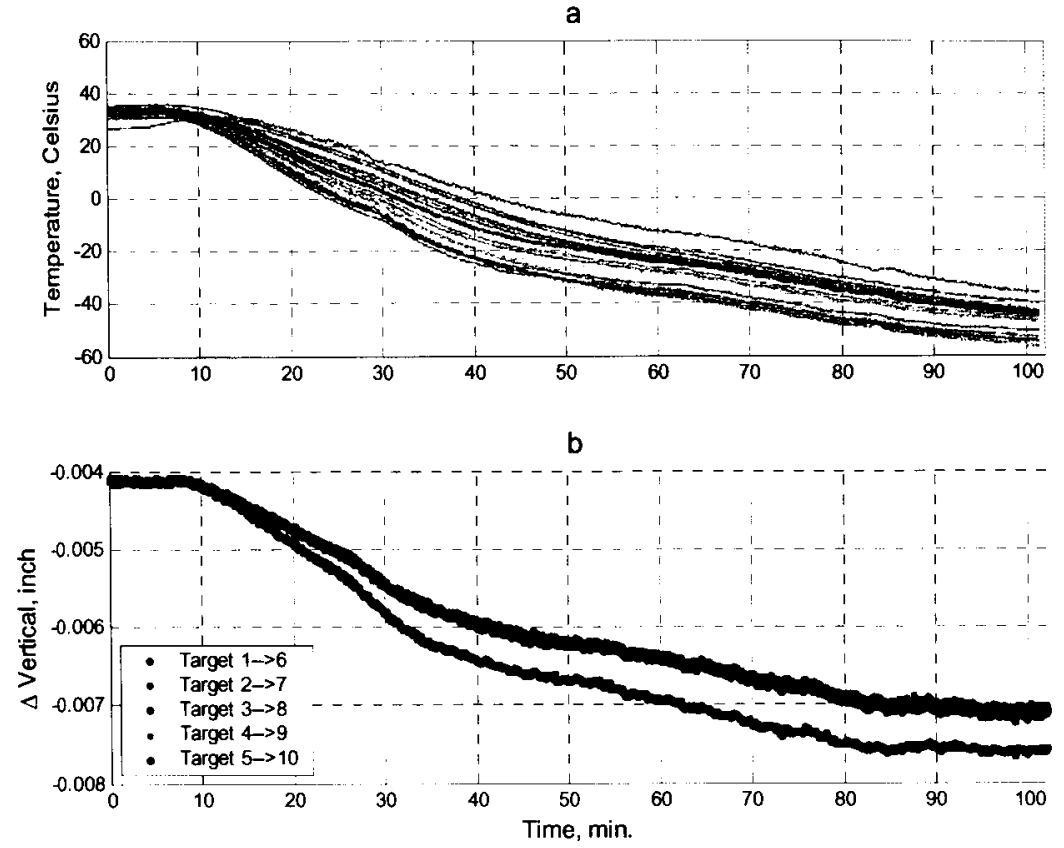

Figure 13. Column temperature profiles (a) and vertical displacement differences between targets sets (1-5) and (6-10) at 41.5 inch separation (b) during cool down prior to a tension test.

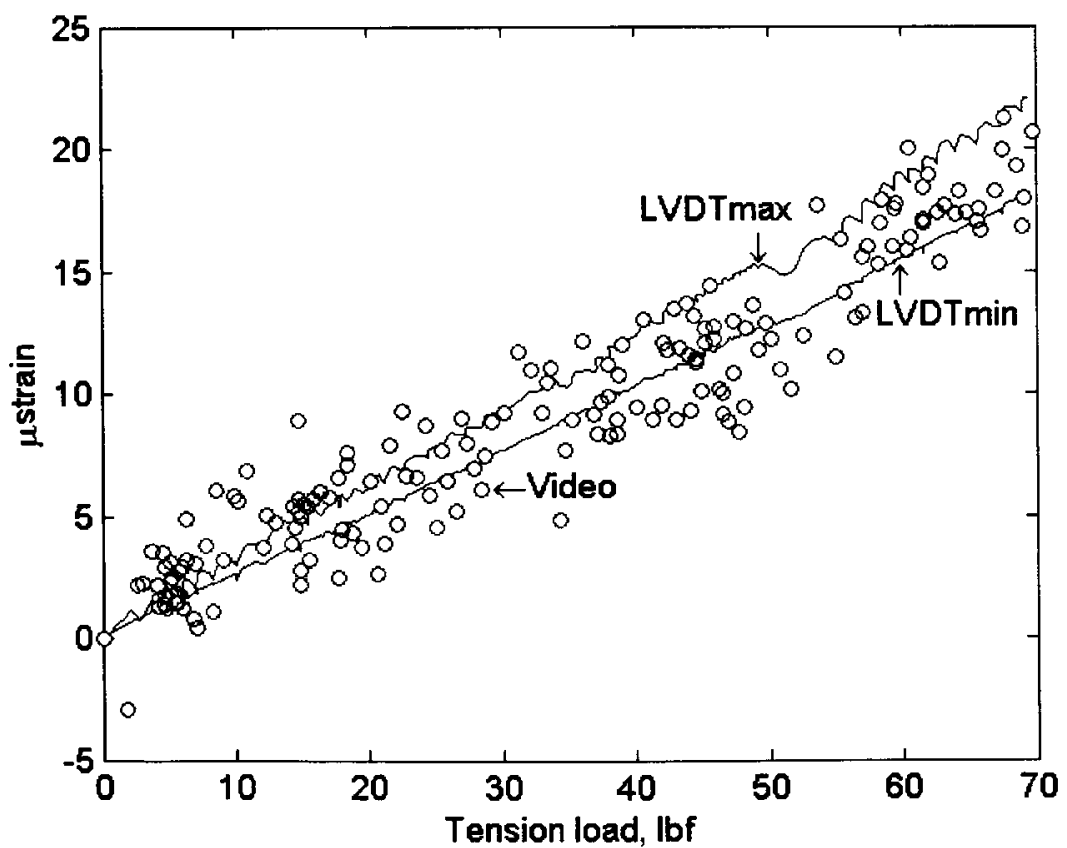

Figure 14. Comparison of video (plotted as circles) and minimum and maximum LVDT $\mu$ strain data (plotted as lines) versus load for a column in tension at $-50^{\circ} \mathrm{C}$. 